attention has been called to it, but in my experience it has proven a helpful and practicable idea.

At the seat of strangulation, circulatory changes begin at once, which will soon be manifested in the serous coat, to the eye; a congestion which deepens and extends further along the distended bowel the longer its exciting cause continues. Should it be some hours, not inches, but feet of the bowel will exhibit a highly colored, injected appearance, which fades from a scarlet at the point of stricture into a pink, and finally is lost in the normal white or gray color. The suggestion is, cautiously bring one loop after another of distended intestine into view at the bottom of the abdominal incision, being directed by the sense of touch to those loops tensely distended, as they are nearest the stricture. By carefully noting their appearance a clew will shortly be found in a loop of congested bowel, and by tracing along in the direction in which the color is deepening the obstruction will be located.

73 Twelfth Street.

\section{HARE LIP, A CASE IN PRACTICE.}

Presented to the Section on Stomatology at the Forty-eighth Annual Meeting of the American Medical Association, beld at Philadelphia, June 1-4, 1897.

BY GEO. T. CARPENTER, M.D., D.D.S.

PROFESSOR OF ORAL SURGERY, COLUMBIAN DENTAI COLLEGR; INSTRUCTOR IN NOSE AND THROAT DEPARTMENT COLIEGE OF PHYSICIANS AND SURGEONS.

CHICAGO. ILL.

The patient, a lady 22 years old, had suffered from birth with double hare lip, also cleft of both hard and soft palate, which was very large, but nature had largely overcome this latter defect by hypertrophy of

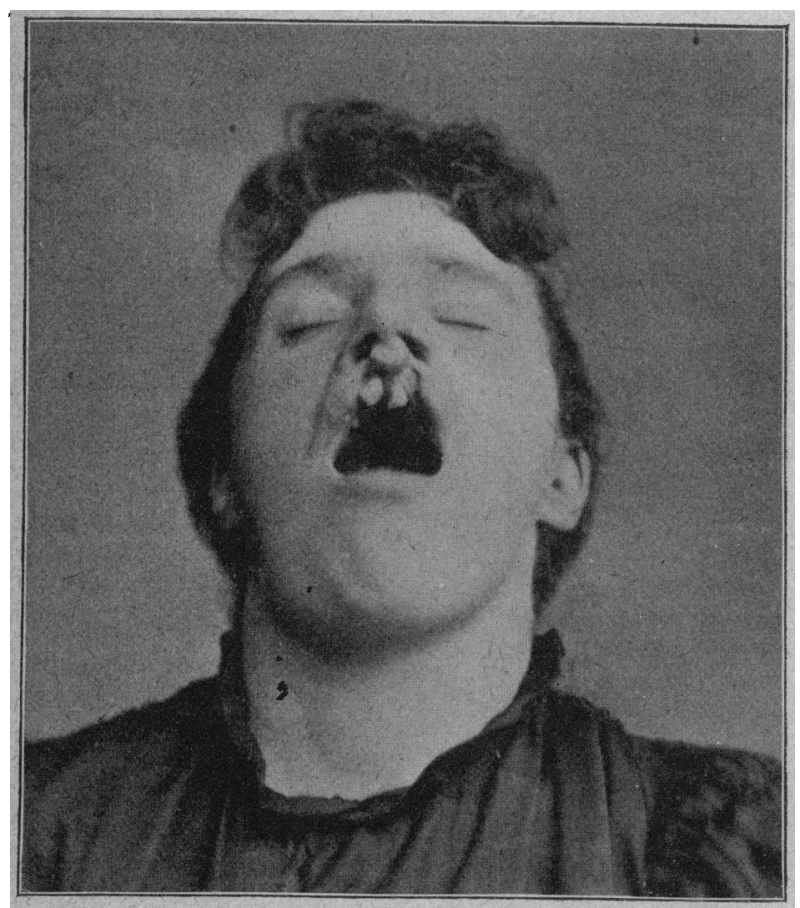

Figure 1.

the inferior turbinated bodies, and, in consequence, the voice, though not good, was much better than many with a very slight opening in the hard palate or bifurcation of the velum. But the deformity of the lip did not improve. The patient was well developed, strong and healthy. She had always lived in the country. Owing to her mother's opposition to surgery the lip was not operated on. The lady was of a healthy and well.dereloped family. She came to Chicago and I was consulted and an appointment made for .July 28, 1896, at which time Figs. 1 and 2 were taken, after which I made a careful examination and found that five anterior teeth were very irregular

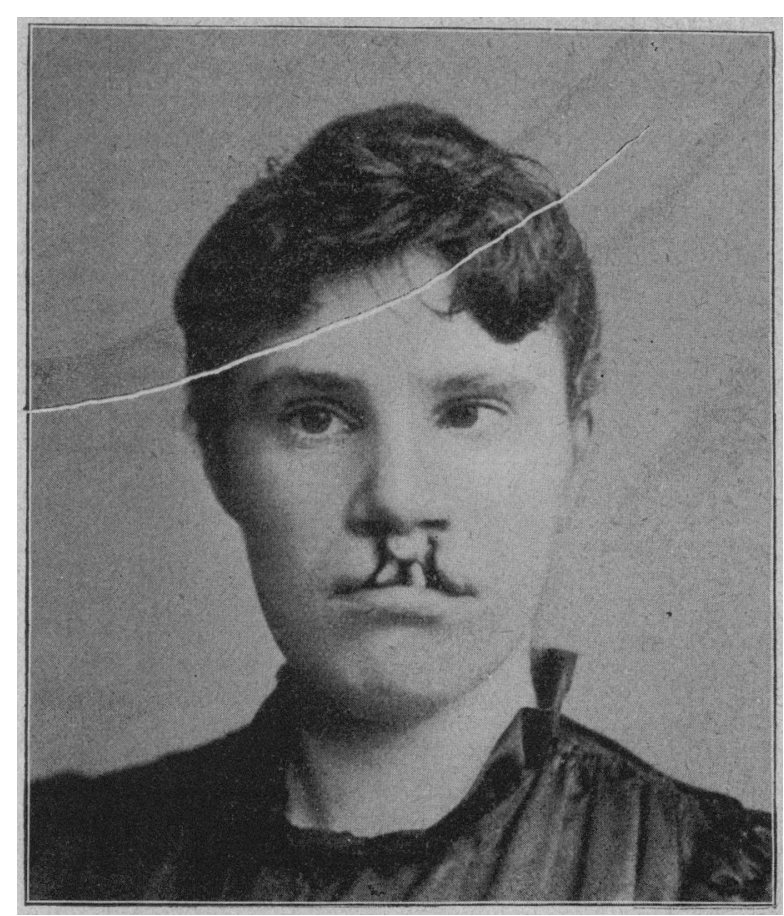

Figure 2.

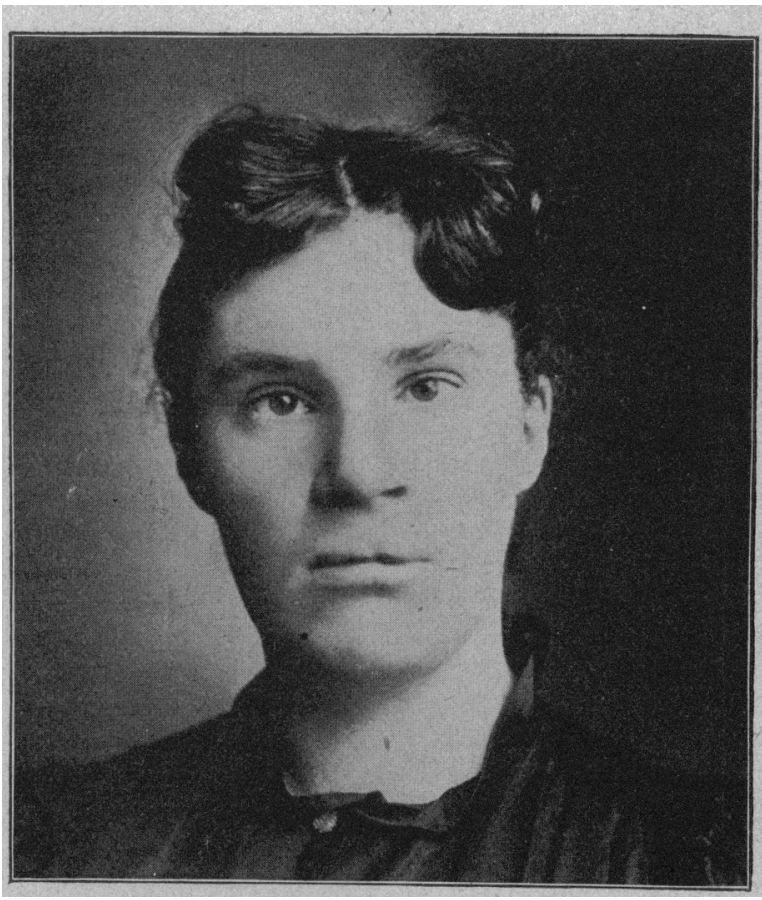

Figure 3.

and badly decayed, the two centrals being movable on account of the loose intermaxillary bones; the cuspids were irregular, but sound. I also took a sectional impression of the upper part of mouth. I then used local applications of 10 per cent. aqueous solution of cocain and removed seven teeth, one of which 
Was supernumerary. As soon as the gums were healed a rabber plate with six anterior teeth and soft rubber velum was inserted, and on September 15 we operated on the lip. The operation was performed under ether. We first removed the mucous membrane, with considerable tissue, from the central teatlike projection. We then passed a lancet through the lip of each side, and cut upward and toward the median line to the extreme upper angle of the cleft. Adhesions of the lip and gum were also freely separated by the use of the knife; then turning the flaps down (the assistant pressing the cheeks well forward), the surfaces of the flaps were brought together. Three deep catgut stitches were taken in the under side of the lip. The margin of the wound was then meatly placed in position and secured by a few fine stitches. Cohesive strips of English moleskin were placed on each cheek, which were allowed to extend to the corners of the mouth and turned under, so as to leave free the anterior ends, which had holes punched for lacing. The surgical dressing was powdered iodoform, covered with 10 per cent. iodoform gauze. The cohesive strips were then laced tightly, so as to bring the cheeks well forward and relieve all tension on the stitches. Dobell's colution was used as a mouth-wash. The lacing was removed and the wound examined each day for a week. The fine stitches were removed the third and deep stitches the sixth day. We had union by first intention. The pationt was dismissed October 12, upon which date the photograph Fig. 3 was taken.

\section{TREATMENT OF CARBUNCLE.}

\section{BY JOHN PHILLIPS, M.D.} STEVENS POINT, WIS.

The Journal of Dec. 12, 1896, p. 1226, contained an article by W. E. Shaw, M.D., of Cincinnati, on the "Conservative Treatment for Carbuncle." My own observations have been so entirely different from the teachings of this article that I am constrained to make a most decided protest against this plan of no treatment, or next to none, of this most formidable disease.

It was the fortune of the writer, in the autumn of 1865 , to suffer from a large and most painful carbuncle, located centrally on the back, overlapping both scapulæ, which was aggravated by attending to professional duties for a week after the disease had made its appearance, not only at home but during the same time making three trips in a buggy a distance of twenty-two miles, returning home in the evening much chilled. By this time I was obliged to go to bed and remain there for the next four weeks, bearing with reasonable fortitude the pain which one writer speaks of as horrible. Gross says: "The pain is throbbing and exceedingly violent, the part feeling as if in contact with melted lead." One who had had personal experience. compared the pain to that of a "large claw. with a hundred talons, grasped into the swollen, inflamed and now exquisitely painful parts, and slowly but constantly tightening its grasp, for days, for weeks, till the last vestige of life is tortured out of the diseased structures." These descriptions of the pain, which may at times be modified in persons and in location, are as nearly correct as any that can be given. Finally recovering, it was with a fixed determination that no patient of mine should suffer is I had with a carbuncle. realized that it was a formidable disease and that little benefit resulted from the measures then being used and believed one would be justified in using more heroic measures in treatment.

In March, 1867, I attended Judge B., 65 years of age, of powerful frame and iron constitution, but considerably broken by hardships and exposures. I found him suffering intensely with a carbuncle of the upper lip. His lip, face, nose and forehead were much swollen. One eye was entirely closed, the other nearly so. A full dose of opium was given at once and pieces of caustic potash were put in two of the three openings in the lip. The potash was pressed well below the surface and the bloody exudation was from time to time carefully wiped away, much care being taken to protect the external surface. The potash was allowed to remain for about twenty minutes, then the wound was well washed out with weakened vinegar, dressed with a warm poultice well wet with tincture of opium, another anodyne was given and the patient dismissed for the night. The next morning all was changed. Nearly all swelling of the lip as well as of the other parts was gone. In reply to my inquiry as to his condition, he said, seizing his lip, "It got well last night; it is a little sore when I pinch it, but it has not given me a bit of pain since an hour after you left me." It did not give him further pain. With dressings of mild cerate it soon healed, leaving but a slight cicatrix.

About two years later I attended the same patient and again found him suffering with a carbuncle, a little above and back of the right hip, but as yet showing no openings. His health was more broken than formerly, having suffered many weeks with sciatica. Dreading the knife, the potash was applied direct to the carbuncle. A plaster of cernte was liberally covered with the caustic and applied, the carbuncle having been first covered with soft leather (buckskin) through which an opening had been made three-fourths of an inch in diameter. The caustic was allowed to remain about forty-five minutes, to make sure of its reaching the deeper diseased structures. The local after-treatment was about the same as at the former time and the results similar. There was no pain except for a short time after the burning. A change, in a few hours, from torture to a quiet and restful condition.

A few months later I attended Barney D., 4 ǒ years of age, much broken in health by exposure and stimulants, who was suffering from a large carbuncle on the back of his neck. The parts were greatly swollen, indurated and exceedingly painful. Anodynes, stimulants and tonics were administered and the caustic ${ }^{1}$ liberally applied, pressing it deep into three of the four openings already existing. This was done notwithstanding the seeming certainty of approaching dissolution. The local after-treatment was similar to that in former cases and the result quite as favorable. The use of the potash was followed by a restful sleep and the same calm hopeful condition.

The next case was that of Mr. C., who was suffering with a large carbuncle just above und a little to the left, but encroaching on the umbilicus. The swelling was large, indurated and exceedingly painful. First making a free incision, the caustic was pressed

1 I usually put a picce of stick caustic alout one-sixth to one-fourth of an ineh in levgth in each opeuryg. In the first ease only laalf thie quantity was used. 